\title{
The evolution of HIV-1 reverse transcriptase in route to acquisition of Q151M multi-drug resistance is complex and involves mutations in multiple domains
}

Jean L Mbisa ${ }^{1 *}$, Ravi K Gupta ${ }^{2}$, Desire Kabamba ${ }^{3}$, Veronica Mulenga ${ }^{3}$, Moxmalama Kalumbi ${ }^{3}$, Chifumbe Chintu ${ }^{3}$, Chris M Parry', Diana M Gibb", Sarah A Walker ${ }^{4}$, Patricia A Cane ${ }^{1}$ and Deenan Pillay ${ }^{1,2}$

\begin{abstract}
Background: The Q151M multi-drug resistance (MDR) pathway in HIV-1 reverse transcriptase (RT) confers reduced susceptibility to all nucleoside reverse transcriptase inhibitors (NRTIs) excluding tenofovir (TDF). This pathway emerges after long term failure of therapy, and is increasingly observed in the resource poor world, where antiretroviral therapy is rarely accompanied by intensive virological monitoring. In this study we examined the genotypic, phenotypic and fitness correlates associated with the development of Q151M MDR in the absence of viral load monitoring.

Results: Single-genome sequencing (SGS) of full-length RT was carried out on sequential samples from an HIVinfected individual enrolled in ART rollout. The emergence of Q151M MDR occurred in the order A62V, V75I, and finally Q151M on the same genome at 4, 17 and 37 months after initiation of therapy, respectively. This was accompanied by a parallel cumulative acquisition of mutations at 20 other codon positions; seven of which were located in the connection subdomain. We established that fourteen of these mutations are also observed in Q151M-containing sequences submitted to the Stanford University HIV database. Phenotypic drug susceptibility testing demonstrated that the Q151M-containing RT had reduced susceptibility to all NRTIs except for TDF. RT domain-swapping of patient and wild-type RTs showed that patient-derived connection subdomains were not associated with reduced NRTI susceptibility. However, the virus expressing patient-derived Q151M RT at 37 months demonstrated $\sim 44 \%$ replicative capacity of that at 4 months. This was further reduced to $\sim 22 \%$ when the Q151Mcontaining DNA pol domain was expressed with wild-type C-terminal domain, but was then fully compensated by coexpression of the coevolved connection subdomain.
\end{abstract}

Conclusions: We demonstrate a complex interplay between drug susceptibility and replicative fitness in the acquisition Q151M MDR with serious implications for second-line regimen options. The acquisition of the Q151M pathway occurred sequentially over a long period of failing NRTI therapy, and was associated with mutations in multiple RT domains.

\section{Background}

RT inhibitors (RTIs) are the mainstay of combination antiretroviral therapy (cART). Recommended first-line therapy regimens for HIV-1 treatment usually comprise two nucleos(t)ide RTIs (NRTIs) plus a third agent, either a non-nucleoside RTI (NNRTI) or a boosted

\footnotetext{
* Correspondence: tamyo.mbisa@hpa.org.uk

'Virus Reference Department, Microbiology Services, Colindale, Health

Protection Agency, London, UK

Full list of author information is available at the end of the article
}

protease inhibitor (bPI) or integrase inhibitor [1-3]. More than 90 mutations have been identified in HIV-1 $\mathrm{RT}$ to be associated with resistance to RTIs, and the majority are clustered either around the polymerase active site or the hydrophobic binding pocket of NNRTIs in the DNA pol domain of RT [4-7]. A consequence of some of these mutations is a severe loss of viral replicative capacity which can subsequently be restored by compensatory mutations elsewhere within RT [8]. 
The Q151M MDR is important because it has been shown to confer resistance to almost all NRTIs with the exception of TDF [9]. The Q151M MDR complex is composed of the Q151M mutation, which is normally the first to appear, followed by at least two of the following four mutations: A62V, V75I, F77L and F116Y [10]. The Q151M MDR complex was initially described to develop during long-term NRTI-containing combination therapy or NRTI therapy with zidovudine (AZT) and/or didanosine (ddI) [11,12]; however, it is now rarely observed in resource-rich countries, where more potent cART is used. It is believed that the Q151M MDR complex occurs infrequently because the Q151 to $M$ mutation requires a 2-bp change (CAG to ATG), and the two possible intermediate changes of Q151L (CAG to CTG) and Q151K (CAG to AAG) significantly reduce viral replication capacity in vitro and are seldom observed in vivo [13-15]. The replicative capacity of a Q151L-containing virus was shown to improve in the presence of S68G and M230I mutations suggesting that compensatory mutations could favour the emergence of the Q151M MDR complex $[13,15]$.

The Q151M complex has been identified in up to $19 \%$ of patients failing therapy containing stavudine $(\mathrm{d} 4 \mathrm{~T})$ as part of ART rollout in the developing world, particularly where treatment is given without virological monitoring, thus allowing long term viraemia whilst on first-line therapy [16-18]. This includes the CHAP2 (Children with HIV Antibiotic Prophylaxis) prospective cohort study of Zambian children on a first-line therapy of lamivudine (3TC)/d4T/nevirapine (NVP) where 2 out of 26 children (8\%) for whom resistance data were obtained had developed resistance via this pathway [19].

Although mutations causing resistance to RTIs have been shown to occur mainly in the DNA pol domain of $\mathrm{RT}$, recent studies have implicated mutations in the Cterminal region of $\mathrm{RT}$ in resistance and possibly in restoring replication fitness of the HIV-1 drug-resistant variants $[20,21]$. Some of these mutations, such as N348I in the connection subdomain, have been reported to have a prevalence of $10-20 \%$ in treatment-experienced individuals [22]. The N348I mutation is associated with M184V and TAMs, and increases resistance to NRTIs such as AZT, as well as the NNRTI NVP. N348I confers resistance by reducing $\mathrm{RNase} \mathrm{H}$ activity which allows more time for the excision or dissociation of the RT inhibitors [22-27]. However, few data are available on the evolution and genetic linkage of C-terminal mutations in the context of Q151M MDR complex, especially in non-B subtypes. In this study, we performed a detailed analysis of sequential samples collected from a patient in the CHAP2 cohort study who had developed resistance via the Q151M pathway to dissect the intrapatient viral population dynamics in the context of full-length RT.

\section{Results}

We investigated the emergence of the Q151M MDR complex in one of the two patients in the CHAP2 cohort study who had developed resistance via the Q151M pathway [19]. The patient, designated P66, was infected with HIV-1 subtype $C$ virus.

\section{Dynamics of emergence and genetic linkage of Q151M MDR complex mutations}

Patients enrolled in the CHAP2 cohort study had CD4 counts done approximately every 6 months and plasma was stored for retrospective viral load and genotypic testing. For patient P66, six samples were collected at 0 , $4,10,17,28$, and 37 months after initiation of therapy; four of which were available for viral load testing and SGS analysis. The viral load and CD4\% counts for patient P66 are shown in figure 1. We initially determined the development of Q151M MDR complex using SGS of full-length RT gene in the four sequential samples collected from patient P66 at 4, 17, 28 and 37 months. More than 30 single-genome sequences were generated per time point except for the 4- and 28month time points when we obtained 6 and 0 sequences respectively. Genetic linkage analysis of the single genomes at 4,17 and 37 months showed that the patient acquired the Q151M MDR mutations in the order: A62V, V75I and finally Q151M (Table 1). The emergence of Q151M after the secondary mutations A62V and V75I is rare. In addition, the analysis showed that drug resistance mutation $\mathrm{T} 69 \mathrm{~N}$ was genetically linked to Q151M MDR mutations and was acquired prior to Q151M.

Accessory mutations in the DNA pol domain of RT have previously been demonstrated in the route to acquisition of Q151M MDR complex in subtype B viruses $[12,28]$. We, therefore, determined whether accessory mutations developed in this subtype C HIV-1 virus and whether the $\mathrm{C}$-terminal region of $\mathrm{RT}$ played a role in the emergence of the Q151M MDR complex. The emergence and presence of mutations in DNA pol domain, connection subdomain and RNase $\mathrm{H}$ domain were assessed by SGS, and their genetic linkage to Q151M MDR mutations was determined. A pre-treatment sample was not available for analysis from patient P66; therefore a codon change was scored as a mutation if it met one of the following criteria: (i) if it was a known drug resistance mutation as determined by International AIDS Society-USA (IAS-USA) [29], (ii) if it was not present in sequences from a previous time point or 


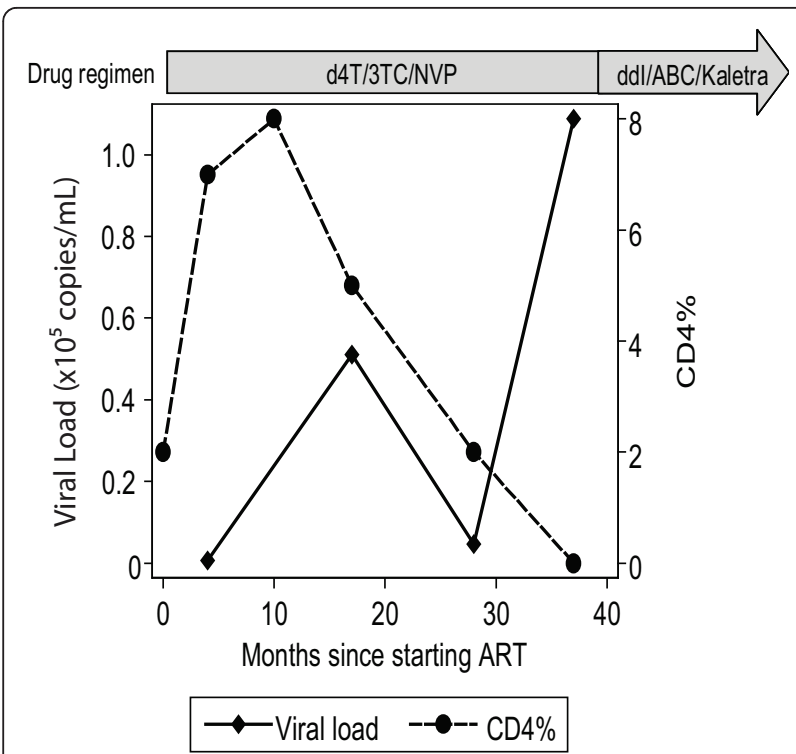

Figure 1 Clinical profile of patient P66. Longitudinal viral load CD4\% and ART regimen data for patient P66 during a 3-year follow up period starting from initiation of CART.

underwent a significant change in frequency between time points. This analysis showed a cumulative increase in mutations in all RT domains (Table 1). Mutations were identified at 12 codon positions in DNA pol domain, namely, $31,33,48,68,102,123,135,174,197$, 202, 203 and 314; seven in connection subdomain, 357, $371,386,399,403,458$ and 471; and one in RNase $H$ domain, 517. The correlation between the progressive increments in the frequency of these mutations and the sequential acquisition of the Q151M MDR mutations suggested that they could be facilitating the emergence of the Q151M MDR complex. This notion is further supported by the observation that 18 out of the 20 mutations were present in a majority of the single genomes by 37 months and nearly half of them were present in all the single genomes (Table 1).

The Q151M MDR mutations were also genetically linked to NRTI mutations M184IV and L210F, and NNRTI mutations E138A, Y181I and H221Y (Table 1). Of note, the N348I mutation was identified in the connection subdomain of all single genomes at 4 months. However, the mutation was present in only one out of 33 single genomes at 17 months but none of the 31 single genomes at 37 months when the Q151M mutation emerged (Table 1).

\section{Intrapatient viral genetic diversity in the route to acquisition of Q151M MDR complex}

The evolution and viral population dynamics within patient P66 were examined further by phylogenetic analyses. Maximum likelihood (ML) trees of the PR-RT single-genome sequences generated from the sequential samples of the patient are shown in Figure 2A. In general, the ML-inferred genealogy clustered all single genomes from each time point within a monophyletic clade with corresponding progressive increases in genetic distances. Intriguingly, the analyses also showed a serial replacement effect with sequences from successive time points arising from a single branch of a cluster of sequences from a preceding time point. This suggests a serial founder effect in the development of Q151M MDR. Furthermore, ML-inferred genealogy of the sequences with drug resistance codons removed showed that the serial founder effect and monophyletic clustering of the sequences from each time point was maintained (Figure 2B). This indicates that the identified accessory mutations could be playing an important role in the evolution and development of the Q151M MDR.

\section{High prevalence of some of the identified accessory mutations in subtype $B$ and $C$ infected patients}

Next, we determined if the 20 accessory mutations that we identified in patient P66 were present in other patients who had developed resistance via the Q151M pathway. We compared mutation frequencies in subtype $\mathrm{B}$ or C samples from RTI-treatment naïve patients and Q151M-containing patient samples on the Stanford University HIV drug resistance database. A significant number of sequences (15 to 12,361) were available for analysis in each subgroup, except for connection subdomain and RNase $\mathrm{H}$ domain of Q151M-containing subtype $C$ sequences, in which there was only one sample sequenced beyond the DNA pol domain. Therefore, the analysis for subtype $C$ sequences could only be carried out for the DNA pol domain. This showed that eight out of the 12 codon positions identified in the DNA pol domain of patient P66 were significantly associated with the sequences containing the Q151M mutation compared to RTI-treatment naïve sequences. These codon positions were 31, 33, 48, 68, 123, 174, 202 and $203(P \leq$ 0.042 ; Table 2$)$. In contrast, two of these codon positions, namely 48 and 174, were not associated with the acquisition of Q151M in subtype B infected patients, but an additional two others were, namely 102 and 197 ( $P \leq$ 0.029). Interestingly, codon positions 386 and 403 in connection subdomain were also significantly associated with the acquisition of Q151M in subtype B infected individuals $(P \leq 0.018)$. These data indicate that some of the accessory mutations identified in the DNA pol domain and connection subdomain of patient P66 are highly prevalent in patients who develop resistance through the Q151M pathway and that they could be playing an important role in the acquisition of the Q151M MDR. 
Table 1 The sequential acquisition of Q151M MDR mutations and the frequency of other RT mutations linked to MDR mutations, in patient P66.

\begin{tabular}{|c|c|c|c|c|c|}
\hline \multirow[t]{3}{*}{ Type or Location of mutations } & \multirow[t]{3}{*}{ Wild-type residue $^{a}$} & \multicolumn{4}{|c|}{ Genetic linkage of other mutations to Q151M MDR } \\
\hline & & \multicolumn{2}{|c|}{4 months $(636)^{b}$} & \multirow{2}{*}{$\begin{array}{c}17 \text { months }(51,000) \\
n=33\end{array}$} & \multirow{2}{*}{$\begin{array}{c}37 \text { months }(108,769) \\
n=31\end{array}$} \\
\hline & & $n=5^{c}$ & $\mathrm{n}=1$ & & \\
\hline & A62 & $\mathrm{V}$ & & $\mathrm{V}$ & $\mathrm{V}$ \\
\hline \multirow[t]{3}{*}{ Q151M MDR } & V75 & & & । & । \\
\hline & Q151 & & & & M \\
\hline & T69 & & & $\mathrm{N}^{45}$ & $\mathrm{~N}^{100}$ \\
\hline \multirow[t]{4}{*}{ Other NRTI } & M184 & $1^{80} \mathrm{~V}^{20 \mathrm{~d}}$ & $1^{100}$ & $V^{100}$ & $V^{100}$ \\
\hline & L210 & & & $S^{6} F^{3}$ & $\mathrm{~F}^{87}$ \\
\hline & V90 & $1^{20}$ & & $1^{3}$ & \\
\hline & E138 & $A^{100}$ & $A^{100}$ & $A^{100}$ & $A^{100}$ \\
\hline \multirow[t]{10}{*}{ NNRTI } & Y181 & $1^{100}$ & $1^{100}$ & $1^{100}$ & $1^{100}$ \\
\hline & $\mathrm{H} 221$ & & & $Y^{70}$ & $Y^{100}$ \\
\hline & M230 & & $L^{100}$ & & \\
\hline & N348 & $1^{100}$ & $1^{100}$ & $1^{3}$ & \\
\hline & 131 & & & $L^{94}$ & $L^{100}$ \\
\hline & A33 & & & & $V^{97}$ \\
\hline & $\mathrm{T} 48$ & & & & $S^{100}$ \\
\hline & S68 & & & & $G^{100}$ \\
\hline & K102 & & & & $R^{61}$ \\
\hline & S123 & & & & $N^{100}$ \\
\hline \multirow[t]{9}{*}{ Other DNA pol domain } & 1135 & $V^{80}$ & & $L^{58} V^{18} T^{15}$ & $T^{100}$ \\
\hline & R174 & & & $\mathrm{K}^{18}$ & $K^{97}$ \\
\hline & K197 & & & & $E^{87}$ \\
\hline & V202 & & & $1^{91}$ & $1^{100}$ \\
\hline & E203 & & & $D^{3}$ & $D^{100}$ \\
\hline & V314 & & & & $1^{26}$ \\
\hline & M357 & & & $R^{18} L^{3}$ & \\
\hline & A371 & & & & $T^{23}$ \\
\hline & T386 & & & $1^{9}$ & $1^{100}$ \\
\hline \multirow[t]{4}{*}{ Other connection subdomain } & E399 & & & $D^{58}$ & $D^{100}$ \\
\hline & A403 & $T^{20}$ & & $T^{45}$ & $T^{97}$ \\
\hline & 1458 & $V^{20}$ & $V^{100}$ & $V^{24}$ & $V^{84}$ \\
\hline & E471 & & & $D^{39}$ & $D^{97}$ \\
\hline RNase $\mathrm{H}$ domain & L517 & $1^{60}$ & $1^{100}$ & $1^{56}$ & $1^{94}$ \\
\hline
\end{tabular}

${ }^{a}$ Wild-type residue was determined based on 4-month sequences and frequency in treatment-naïve individuals as determined using Stanford University HIV database

${ }^{\text {b }}$ Viral load in copies $/ \mathrm{mL}$

c Number of single genomes linked or unlinked to Q151M MDR mutations

d Percent of single genomes with that particular mutation calculated as follows: number of mutations per codon/number of single genomes linked or unlinked to Q151M MDR (n) $\times 100 \%$

C-terminal mutations are not associated with decreased susceptibility of Q151M-containing viruses to NRTIs in patient P66

Consequently, we investigated whether the C-terminal mutations we observed affected susceptibility to NRTIs. Unique restriction sites were introduced in RT and IN genes without changing the amino acid coding, in both the packaging vector and cloned patient fragments in order to facilitate RT domain-swapping (Figure 3A). The patient-derived RTs remained $\mathrm{d} 4 \mathrm{~T}$-susceptible until the development of the Q151M mutation at 37 months, when there was a significant increase ( 16-fold) in $\mathrm{IC}_{50}$ values compared to wild-type RT (Figure 3B; $P<0.002$ ). At most we observed a 1.3-fold change in susceptibility to $\mathrm{d} 4 \mathrm{~T}$ at 4 or 17 months leading us to conclude that Q151M is the main contributor to $\mathrm{d} 4 \mathrm{~T}$ resistance in the Q151M MDR complex. The patient-derived RT exhibited a 23 -fold increase in $3 \mathrm{TC} \mathrm{IC}_{50}$ values at 4 months which did not increase at 17 and 37 months despite the acquisition of the Q151M MDR mutations (Table 3). 


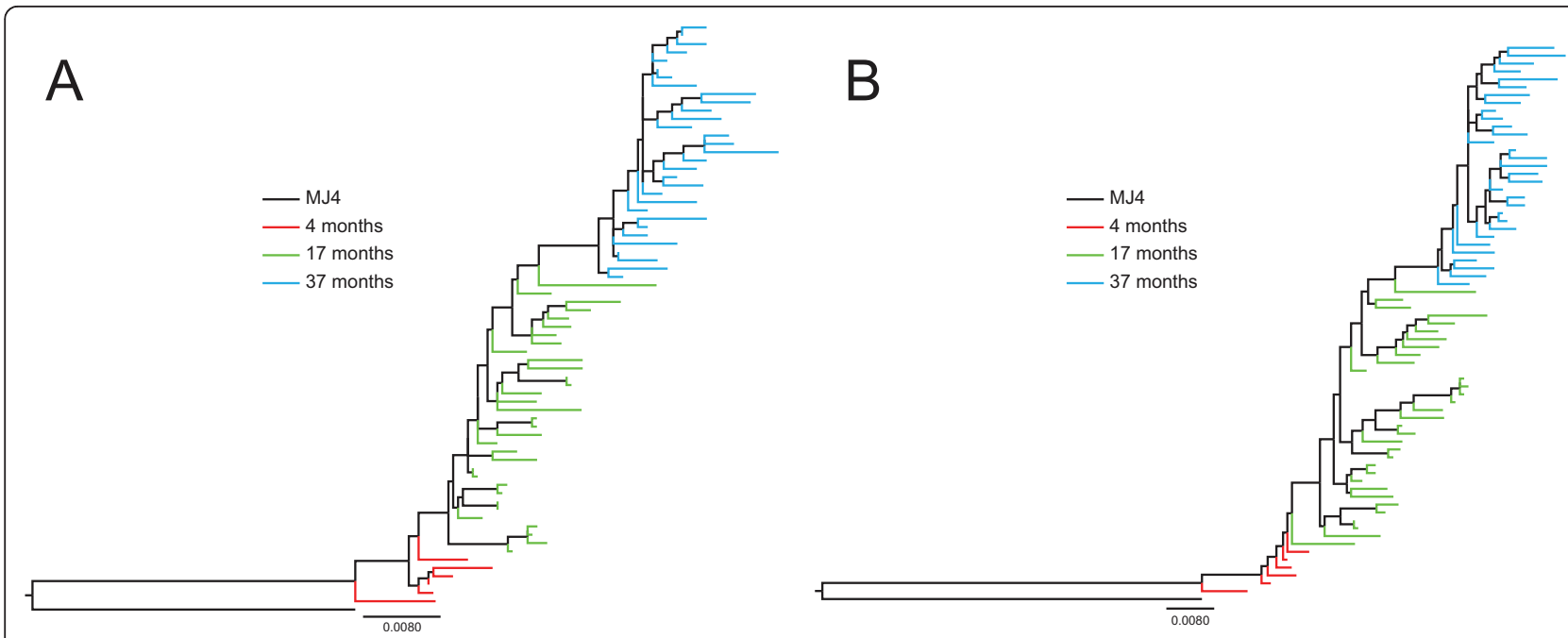

Figure 2 ML phylogenetic analysis of single genome sequences. Branch lengths were estimated using the GTR model of substitution and are drawn in scale with the bar at the bottom representing 0.008 nucleotide substitutions per site. The colour of each tip branch represents the time after initiation of therapy when the sample from which the single-genome originates was collected as shown in the legend in each figure. (A) Phylogenetic tree of 70 single genomes generated from 3 sequential samples from patient P66 infected with subtype C HIV-1 virus. (B) Same as (A) but with the following 12 RT drug resistance codons removed from the aligned single-genome sequences to determine the effect of drug resistance mutations on viral evolution: $62,69,75,90,138,151,181,184,210,221,230$ and 348 . The trees were rooted using the subtype $C$ reference sequence MJ4.

The effect on susceptibility to $3 \mathrm{TC}$ was probably due to M184I/V mutations which were seen by 4 months. The 23 -fold reduction in susceptibility is relatively lower than observed in other studies [30,31]. This could be because our assay uses full-length RT fragments derived from clinical isolates. It has recently been shown that the use of a co-evolved or subtype-specific C-terminal region of RT can influence the magnitude of drug resistance observed in a phenotypic drug susceptibility assay [32].

Analysis of susceptibilities of patient-derived RTs to the CHAP2 second-line NRTIs ddI and ABC showed a cumulative decrease in susceptibility in the order; 1.2 and 1.7-fold at 4 months, 4- and 6-fold at 17 months, and finally 9.9- and 10.8-fold at 37 months, respectively (Figure 3C). Thus, unlike $\mathrm{d} 4 \mathrm{~T}$ the cumulative acquisition of mutations on the route to Q151M MDR complex results in a parallel cumulative decrease in susceptibilities to $A B C$ and ddI. In addition, the recombinant viruses expressing patient-derived RTs exhibited decreased susceptibilities to NRTIs FTC of $>79$-fold at 4 months and AZT of $>15$-fold at 37 months (Table 3 ) but remained susceptible to TDF even after the acquisition of the Q151M mutation at 37 months (Figure 3D) with no significant increases in $\mathrm{IC}_{50}$ values $(P>0.18)$. The susceptibility to TDF could probably be influenced by the presence of $\mathrm{M} 184 \mathrm{~V}$ which has been shown to increase HIV-1 sensitivity to TDF [33,34].

The expression of the patient-derived DNA pol domain at 37 months plus wild-type $\mathrm{C}$-terminal region or coevolved connection subdomain showed no significant differences in $\mathrm{IC}_{50}$ values to $\mathrm{d} 4 \mathrm{~T}(P>0.05)$ suggesting that none of the identified $\mathrm{C}$-terminal mutations in patient $\mathrm{P} 66$ at 37 months contributed to the reduction in susceptibility to d4T (Figure 3B). Similarly, the coevolved C-terminal region did not contribute to $3 \mathrm{TC}$ resistance, including the previously identified N348I mutation at 4 months, neither did they contribute to the decreases in susceptibility to ABC, ddI or FTC (Figure $3 \mathrm{C}$ and $3 \mathrm{D}$ and Table 3 ). However, we observed an effect of the C-terminal mutations at 37 months to $\mathrm{AZT}$, with the co-evolved C-terminal region contributing a 2.5-fold increase in AZT resistance (Table 3).

Finally, we determined the effect of the mutations on susceptibility to NVP, the NNRTI used for first-line therapy in the CHAP2 cohort study. The recombinant viruses expressing the patient-derived $\mathrm{C}$-terminal region at 4 months, but not at 17 or 37 months, exhibited a 5fold increase in the NVP $\mathrm{IC}_{50}$ value relative to wild-type $(P<0.002$; Table 4$)$. The decrease in NVP susceptibility associated with the $\mathrm{C}$-terminal domain at 4 months is likely due to the presence of the N348I mutation in the connection subdomain which disappears at later time points.

\section{Connection subdomain mutations in patient P66 partially restore replicative fitness of Q151M MDR-containing viruses}

Since we did not observe any association of C-terminal mutations at 37 months with a decrease in susceptibilities 
Table 2 Analysis of the frequency of accessory mutations in RTI-treatment naïve and Q151M-containing sequences on Stanford University HIV database.

\begin{tabular}{|c|c|c|c|c|c|c|c|c|c|c|c|c|}
\hline \multirow[b]{3}{*}{$\begin{array}{l}\text { RT } \\
\text { domain }\end{array}$} & \multicolumn{6}{|c|}{ Subtype C } & \multicolumn{6}{|c|}{ Subtype B } \\
\hline & \multirow[b]{2}{*}{$\begin{array}{l}\text { Wild- } \\
\text { type } C^{\text {a }}\end{array}$} & \multicolumn{2}{|c|}{ RTI-treatment naïve } & \multicolumn{2}{|c|}{ Q151M ${ }^{b}$} & \multirow[b]{2}{*}{$\begin{array}{l}\text { Mut.\% } \\
\text { Diff. }^{\text {e }}\end{array}$} & \multirow[b]{2}{*}{$\begin{array}{l}\text { Wild- } \\
\text { type B }\end{array}$} & \multicolumn{2}{|c|}{$\begin{array}{l}\text { RTI-treatment } \\
\text { naïve }\end{array}$} & \multicolumn{2}{|c|}{ Q151M } & \multirow[b]{2}{*}{$\begin{array}{l}\text { Mut.\% } \\
\text { Diff. }\end{array}$} \\
\hline & & $\begin{array}{l}\text { No. of } \\
\text { seqs.c }^{c}\end{array}$ & $\begin{array}{l}\% \text { mut. } \\
\text { freq. }{ }^{d}\end{array}$ & $\begin{array}{l}\text { No. of } \\
\text { seqs. }\end{array}$ & $\begin{array}{l}\% \text { mut. } \\
\text { freq. }\end{array}$ & & & $\begin{array}{l}\text { No. of } \\
\text { seqs. }\end{array}$ & $\begin{array}{l}\% \text { mut. } \\
\text { freq. }\end{array}$ & $\begin{array}{l}\text { No. of } \\
\text { seqs. }\end{array}$ & $\begin{array}{l}\% \text { mut. } \\
\text { freq. }\end{array}$ & \\
\hline & 131 & 3,557 & $<1$ & 24 & $4(\mathrm{~L})$ & +4 & 131 & 10,329 & $<1$ & 373 & $5(\mathrm{RL})$ & +5 \\
\hline & A33 & 3,600 & $<0.1$ & 24 & $4(V)$ & +4 & A33 & 10,388 & $<1$ & 375 & $2(V)$ & +2 \\
\hline & T48 & 3,941 & 15 (SE) & 44 & 39 (S) & +24 & S48 & 12,361 & $3(T)$ & 492 & $2(T)$ & -1 \\
\hline & S68 & 3,998 & $<1$ & 44 & $73(\mathrm{G})$ & +73 & S68 & 12,350 & $4(\mathrm{G})$ & 491 & $\begin{array}{c}50 \\
\text { (GNRK) }\end{array}$ & +46 \\
\hline & K102 & 4,004 & $2(\mathrm{Q})$ & 44 & $5(\mathrm{QN})$ & +3 & K102 & 12,204 & $5(\mathrm{QR})$ & 492 & $8(\mathrm{QR})$ & +3 \\
\hline & D123 & 3,757 & 62 (SGNE) & 44 & 77 (SGN) & +15 & D123 & 12,001 & 29 (ENS) & 492 & 28 (EN) & -1 \\
\hline \multirow[t]{9}{*}{ DNA pol } & 1135 & 3,942 & 28 (TVR) & 44 & $\begin{array}{c}23 \\
\text { (TVMK) }\end{array}$ & -5 & 1135 & 11,994 & 43 (TVLR) & 492 & $\begin{array}{c}38 \\
\text { (TVLMR) }\end{array}$ & -5 \\
\hline & Q174 & 3,851 & 39 (KR) & 44 & 61 (KR) & +22 & Q174 & 12,241 & 7 (KEHR) & 492 & 9 (RKH) & +2 \\
\hline & Q197 & 3,999 & $3(K)$ & 44 & $2(E)$ & -1 & Q197 & 12,316 & 3 (KE) & 492 & 5 (EK) & +2 \\
\hline & 1202 & 3,955 & $7(V)$ & 44 & $27(V)$ & +20 & 1202 & 12,151 & $9(V)$ & 492 & $24(V)$ & +15 \\
\hline & E203 & 4,008 & 1 & 44 & $7(K)$ & +6 & E203 & 12,304 & 1 & 492 & 10 (DK) & +9 \\
\hline & V314 & 1,889 & $2(A)$ & 19 & 0 & -2 & V314 & 4,332 & $<1$ & 91 & 0 & 0 \\
\hline & M357 & 715 & $\begin{array}{c}33 \\
(\mathrm{RKLVIT})\end{array}$ & 1 & $100(K)$ & $N C^{f}$ & M357 & 1,481 & 31 (TKVIR) & 75 & 33 (TVRKI) & +2 \\
\hline & A371 & 684 & $6(V)$ & 1 & 0 & NC & A371 & 1,518 & $5(\mathrm{~V})$ & 75 & $11(\mathrm{VT})$ & +6 \\
\hline & T386 & 657 & $11(\mathrm{IV})$ & 1 & $100(\mathrm{I})$ & $\mathrm{NC}$ & T386 & 1,504 & 18 (IV) & 75 & $\begin{array}{c}49 \\
\text { (IAVSPM) }\end{array}$ & +31 \\
\hline \multirow[t]{4}{*}{ connection } & E399 & 595 & $5(\mathrm{DG})$ & 1 & 0 & NC & E399 & 1,381 & $14(\mathrm{D})$ & 75 & $13(\mathrm{DG})$ & -1 \\
\hline & T403 & 556 & 6 (MASI) & 0 & $N A^{g}$ & NA & T403 & 744 & $\begin{array}{c}23 \\
\text { (MISAVL) }\end{array}$ & 17 & 0 & -23 \\
\hline & V458 & 401 & $6(I)$ & 0 & NA & NA & V458 & 651 & $1(\mathrm{I})$ & 16 & 0 & -1 \\
\hline & E471 & 396 & $3(D)$ & 0 & NA & NA & D471 & 658 & 3 (EN) & 16 & 0 & -3 \\
\hline RNase $\mathrm{H}$ & L517 & 392 & $7(I)$ & 0 & NA & NA & L517 & 636 & 15 (IV) & 15 & 0 & -15 \\
\hline \multirow{2}{*}{\multicolumn{13}{|c|}{$\begin{array}{l}\text { aThe residue occurring in the majority of RTI-t } \\
\text { difference in mutation frequency between RTI } \\
0.024), \text { T48 }(P<0.0001), \mathrm{S} 68(P<0.0001), \mathrm{D} 12 \\
\text { S68 }(P<0.0001), \mathrm{K} 102(P=0.006), 1135(P=0 \\
\text { bS } S \text { Sequences containing the Q151M mutation }\end{array}$}} \\
\hline & & & & & & & & & & & & \\
\hline \multicolumn{13}{|c|}{ 'The number of sequences used for the analysis. Only one sequence was used per individual if multiple sequences were available. } \\
\hline \multicolumn{13}{|c|}{$\begin{array}{l}{ }^{\mathrm{d}} \text { The percentage of sequences with an amino acid change from wild-type residue. The mutant amino acid(s) present at a frequency greater than } 1 \% \text { is shown in } \\
\text { brackets. }\end{array}$} \\
\hline $\begin{array}{l}{ }^{e} \text { The differen } \\
\text { in mutation } \mathrm{f}\end{array}$ & $\begin{array}{l}\text { e in mutatio } \\
\text { equency in }\end{array}$ & $\begin{array}{l}\text { n frequenc } \\
\text { 2151M-con }\end{array}$ & $\begin{array}{l}\text { between } \mathrm{Q} 151 \\
\text { ining sequenc }\end{array}$ & $\begin{array}{l}\text { M-contain } \\
\text { es compar }\end{array}$ & $\begin{array}{l}\mathrm{ig} \text { and RTI-t } \\
\mathrm{d} \text { to RTI-trea }\end{array}$ & $\begin{array}{l}\text { atment na } \\
\text { ment naïve }\end{array}$ & e sequence & plus sign & indicates an & crease ar & minus sign & decrease \\
\hline$c=$ Not ca & culated (one & sequence & railable for ana & & & & & & & & & \\
\hline
\end{tabular}

to first-line drugs, we evaluated their effect on virus replicative capacity by infecting HEK293T cells with equivalent amounts of virus. The patient's sample before initiation of therapy was not available, thus the replicative capacity of the viruses measured by relative luciferase light units was compared to that of the virus expressing full-length patient-derived RT at 4 months. The patientderived RT at 4 months had already developed the M184I mutation which is known to affect viral replicative fitness $[35,36]$. The virus expressing the full-length patient-derived RT containing the Q151M mutation at
37 months demonstrated $\sim 42 \%$ replicative capacity of full-length patient-derived RT at 4 months $(P<0.0001$; Figure 2E). This was further significantly decreased to $\sim 22 \%(P<0.0001)$ when the patient-derived DNA pol domain at 37 months was expressed in combination with wild-type connection subdomain and RNase $\mathrm{H}$ domain. This decrease in replicative capacity was fully compensated (to $\sim 55 \%$ replicative capacity) by the coexpression of the coevolved connection subdomain at 37 months. In contrast, replicative capacity of the full-length patientderived RT at 17 months was comparable to that at 4 


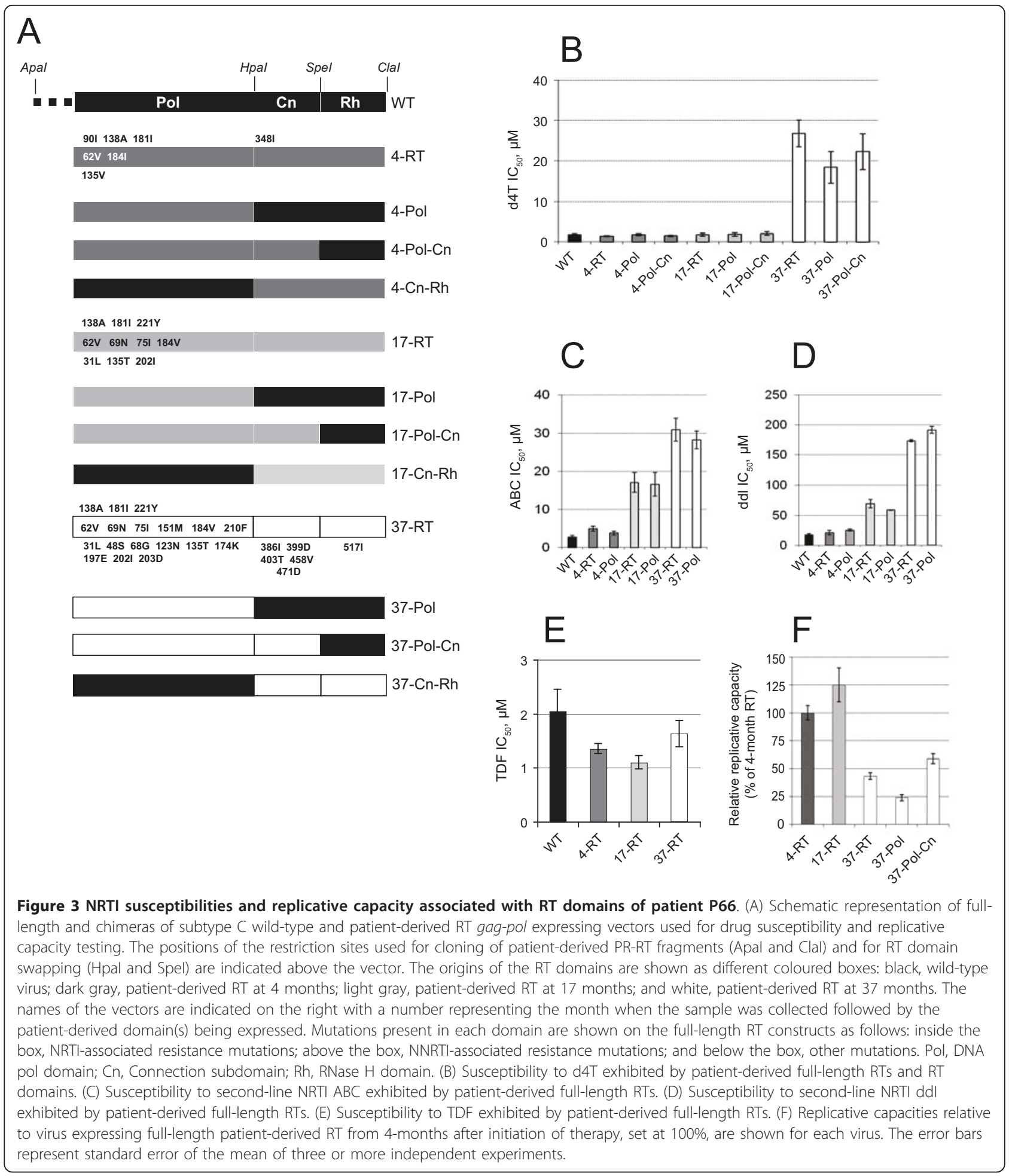

months. This suggests that the Q151M mutation, as well as being the main determinant of drug resistance in the Q151M MDR complex, also has a more significant effect on virus replication fitness that is partially restored by mutations in the connection subdomain.

\section{Discussion}

Multiple mutations throughout HIV-1 RT are associated with RTI resistance including recently identified mutations in the connection subdomain and RNase $\mathrm{H}$ domain $[10,21,27]$. However, there are few data on 
Table 3 3TC, AZT and FTC susceptibilities associated with RT domains of patient P66.

\begin{tabular}{ccccccc}
\hline Virus & \multicolumn{2}{c}{ 3TC } & \multicolumn{2}{c}{ AZT } & \multicolumn{2}{c}{ FTC } \\
\cline { 2 - 7 } & $\mathbf{I C}_{\mathbf{5 0}}{ }^{\mathbf{a}}$ & $\mathbf{F C}^{\mathbf{b}}$ & $\mathbf{I C}_{\mathbf{5 0}} \mathbf{a}^{\mathbf{a}}$ & $\mathbf{F C}^{\mathbf{b}}$ & $\mathbf{I C}_{\mathbf{5 0}} \mathbf{a}^{\mathbf{2}}$ & $\mathbf{F C}^{\mathbf{b}}$ \\
\hline $\begin{array}{c}\text { Wild- } \\
\text { type }\end{array}$ & $8.5 \pm 0.8$ & & $168.6 \pm 46.8$ & & $2.2 \pm 0.3$ & \\
4-RT & $198.8 \pm 18.6$ & 23.3 & $76.9 \pm 6.8$ & 0.5 & $184.2 \pm 14.2$ & 84.9 \\
4-Pol & $211.5 \pm 17.5$ & 24.8 & $60.0 \pm 13.8$ & 0.4 & $168.1 \pm 6.4$ & 77.5 \\
17-RT & $224.1 \pm 16.9$ & 26.3 & $56.4 \pm 7.2$ & 0.3 & $228.8 \pm 6.7$ & 105.5 \\
17-Pol & $206.5 \pm 9.7$ & 24.2 & $58.1 \pm 14.2$ & 0.3 & $218.9 \pm 13.3$ & 100.9 \\
37-RT & $219.7 \pm 7.5$ & 25.8 & $5120.9 \pm 515.6$ & 30.4 & $230.9 \pm 10.2$ & 106.4 \\
37-Pol & $217.8 \pm 18.1$ & 25.6 & $2025.3 \pm 144.2$ & 12.0 & $231.5 \pm 17.1$ & 106.7 \\
\hline
\end{tabular}

${ }^{\mathrm{a}} 50 \%$ inhibitory concentration in $\mathrm{nM} \pm \mathrm{SEM}$.

${ }^{\mathrm{b}}$ Fold change in $\mathrm{IC}_{50}$ compared to wild-type virus.

sequential acquisition and genetic linkage of these mutations and their impact on drug susceptibility and replicative capacity, especially in non-B subtype HIV-1 viruses which account for nearly $90 \%$ of the epidemic worldwide [37]. In this study, we took advantage of treatment failure in the absence of viral load-guided therapy to dissect the relative contribution of RT domains in the route to high-level NRTI drug resistance through the Q151M pathway.

As expected we found that the development of mutations was broad throughout RT. The virus from the patient we investigated had developed more than 12 known drug resistance mutations and 20 additional mutations in RT, nearly half of which were located in the connection subdomain. A refined analysis of the emergence and development of these mutations in sequential samples by SGS revealed a chronological increase in frequency that paralleled the sequential acquisition of Q151M MDR mutations. In addition, the

Table 4 NVP susceptibilities associated with RT domains of patient P66.

\begin{tabular}{ccc}
\hline Virus & $\mathrm{IC}_{\mathbf{5 0}}{ }^{\mathbf{a}}$ & $\mathrm{FC}^{\mathbf{b}}$ \\
\hline Wild-type & $86.47 \pm 11.84$ & \\
\hline $4-\mathrm{RT}$ & $>6,000$ & $>66$ \\
$4-\mathrm{Pol}$ & $>6,000$ & $>66$ \\
$4-\mathrm{Pol}-\mathrm{Cn}$ & $>6,000$ & $>66$ \\
4-Cn-Rh & $410.5 \pm 55.2$ & 4.7 \\
\hline 17-RT & $>6,000$ & $>66$ \\
17-Pol & $>6,000$ & $>66$ \\
17-Pol-Cn & $>6,000$ & $>66$ \\
17-Cn-Rh & $73.83 \pm 8.54$ & 0.9 \\
\hline 37-RT & $>6,000$ & $>66$ \\
37-Pol & $>6,000$ & $>66$ \\
37-Pol-Cn & $>6,000$ & $>66$ \\
37-Cn-Rh & $88.23 \pm 12.95$ & 1.0
\end{tabular}

${ }^{\mathrm{a}} 50 \%$ inhibitory concentration in $\mathrm{nM} \pm \mathrm{SEM}$.

${ }^{\mathrm{b}}$ Fold change in $\mathrm{IC}_{50}$ compared to wild-type virus. analysis showed genetic linkage of most of these mutations to Q151M MDR mutations indicating an association between the two. Although our results are from one patient, the identified mutations in the pol domain at codon positions 68 and 202 were previously identified in patients infected with subtype B HIV-1 viruses $[12,28]$ and in an HIV database sequence analysis done in this study (Table 2). The database sequence analysis also showed that the DNA pol domain mutations at codon positions 31, 33, 48, 102, 123, 135, 174, 197 and 203 were significantly associated with Q151M in subtype $\mathrm{B}$ and/or C.

We show that although the connection subdomain mutations were acquired in parallel with Q151M MDR mutations they were not directly associated with drug resistance but played a role in improving the replicative fitness of the Q151M-containing viruses. Our findings confirm previous reports showing that the Q151M-containing virus replicates poorly $[13,14,38,39]$. We clearly show that the patient-derived connection subdomain is important for improving the Q151M-containing virus' replicative fitness and is thus important for the development of the Q151M pathway. It will be interesting to elucidate the particular mutations involved and the mechanism behind the connection subdomain's effect on replicative fitness of the Q151M-containing RT. The mutation at connection subdomain codon positions 386 and 403 were significantly associated with Q151M in the subtype B database analysis; however, a similar analysis could not be carried out for subtype $C$ due to lack of samples sequenced beyond the DNA pol domain. Since the connection subdomain is involved in positioning of the template-primer complex at the polymerase active site, one possibility could be that the mutations improve enzyme-substrate interactions at the active site. Of note, the intermediate Q151K or L mutations which have been postulated to be involved in the emergence of the Q151M mutation were never identified in our SGS analysis. It is possible that these mutations do emerge but are only present transiently due to their negative effect on replication and, as a result, were missed in this analysis. This possibility could not be explored further in this study as we were unable to amplify any genomes at 28 months, the time point prior to the emergence of the Q151M mutation.

It was surprising to observe that the patient-derived connection subdomain and RNase $\mathrm{H}$ domain were not associated with the decreased susceptibility to NRTIs exhibited by the Q151M MDR-containing RTs and also that the N348I mutation disappeared prior to the acquisition of Q151M. As described earlier, N348I confers drug resistance by decreasing $\mathrm{RNase} H$ activity, thus it will be interesting to explore if a negative correlation exists between reduced RNase $\mathrm{H}$ activity and Q151M. 
Another surprising finding was that full-blown resistance did not develop until 37 months after initiation of therapy, even though the viral load had been relatively high at earlier time points. This raises the possibility of suboptimal use of the drugs contributing to the emergence of the Q151M MDR complex.

\section{Conclusions}

Understanding the evolution and molecular mechanisms leading to the emergence of the Q151M MDR complex is important especially in light of its relatively frequent occurrence in some ARV rollout cohorts. As shown in this study and other previous reports [9], the presence of the Q151M mutation significantly limits the options for second-line therapies as the Q151M-containing virus remains only susceptible to one approved NRTI, TDF. Our results showed that the Q151M MDR takes a long time to develop and keeping patients on failing NRTI therapy could be facilitating its emergence. The Q151M MDR is also often linked to other NRTI and NNRTI mutations which develop earlier and thus further limiting the options for second-line regimens. In addition, the virus acquires compensatory mutations throughout RT which make it fitter, resulting in a virus that could persist even after switching to second-line therapy. This is a major obstacle in the developing world where fixed second-line therapies are composed of two alternate NRTIs (usually not TDF) and bPI. Thus, these types of studies are important in guiding public health approaches to the treatment and clinical management of HIV-1 infections in resource-poor settings.

\section{Methods}

\section{Clinical HIV samples and database analysis}

The plasma samples characterized in this study were from a patient enrolled in the CHAP2 prospective cohort study at the University Teaching Hospital in Lusaka, Zambia [19]. Children in this study were initiated on first-line cART of $3 \mathrm{TC} / \mathrm{d} 4 \mathrm{~T} / \mathrm{NVP}$ (adult Triomune30) and, following immunological or clinical failure, were switched to a fixed second-line therapy of Abacavir (ABC)/ddI/Kaletra. The prevalence of identified accessory mutations in clinical samples was analyzed using the Stanford University HIV drug resistance database (http://hivdb.Stanford.edu).

\section{SGS assay}

A previously described SGS assay [40] was modified by designing new antisense primers in integrase (IN) and used to sequence the full-length protease (PR) and RT genes from sequential samples. Briefly, viral RNA was extracted from $200 \mu \mathrm{L}$ of plasma using QIAmp UltraSens Virus Kit (Qiagen) following manufacturer's instructions and eluted in $60 \mu \mathrm{L}$ of elution buffer.
cDNA synthesis and single genome PCR reactions were carried out as described previously [40] using primers 1849+ (5'-GATGACAGCATGTCAGGGAG-3') and 4368- (5'-GCTAGCTACTATTTCTTTTGCTACT-3'), followed by a nested PCR with primers $1870+\left(5^{\prime}-\right.$ GAGTTTTGGCTGAGGCAATGAG-3') and 4295- (5'CTTTCATGCTCTTCTTGAGCCT-3'). Positive PCR products were identified by agarose gel electrophoresis and purified using illustra GFX PCR DNA and Gel Band Purification Kit (GE Healthcare), and sequenced by the dideoxy ABI sequencing systems in both directions using overlapping internal primers. Sequences were analyzed using Sequencher software (Gene Codes) and aligned by using subtype-specific consensus sequences. Any sequences containing double peaks in the chromatographs were excluded. Drug resistance mutations were defined by using the Stanford University HIV drug resistance database.

\section{Phylogenetic analyses}

Full-length PR-RT nucleotide single-genome sequences from patient $\mathrm{P} 66$ and subtype-specific reference sequence MJ4 (subtype C) were aligned using Clustal W in MEGA4 software [41]. The aligned sequences were imported into PhyML tree building software and ML trees were constructed using the GTR model and the robustness of the trees was evaluated by bootstrap analysis with 500 rounds of replication.

\section{Single-replication cycle drug susceptibility assay}

A recently described three plasmid-based retroviral vector system using a luciferase reporter gene was used to study phenotypic drug susceptibility $[42,43]$. Briefly, vector p8MJ4 was modified to accommodate RT domainswapping by introducing three restriction enzyme sites, HpaI (flanking RT amino acids 288/289), SpeI (flanking RT amino acids 423/424) and ClaI (flanking IN amino acids 4/5) creating p8MJ4-HSC. The MJ4 sequence also contains a natural and unique ApaI site in p6 region of gag. In addition, the SpeI site in gag and two ClaI sites (upstream of gag initiation codon and in gag) were eliminated to ensure that the introduced sites were unique. In parallel, patient-derived PR-RT single genomes that closely represented the sequence of the majority of the single genomes at each time point were subcloned into a TOPO-TA vector (Invitrogen) by PCR using primers GagApaF (5'-GCAGGGCCCCTAGGAAAAAGGGC-3') and CRhINClaIR1 (5'-CCTTATCGATTCCATCTAGAAATAGC-3'). Similarly, HpaI (flanking RT amino acids 288/289) and SpeI (flanking RT amino acids 423/424) sites were introduced and any $\mathrm{HpaI}$ or SpeI sites that were present in the cloned patient fragments were removed using sequence-specific primers. Mutagenesis reactions were carried out by site- 
directed mutagenesis using QuikChange Lightning Multi Site-Directed Mutagenesis Kit (Agilent Technologies) and the presence and absence of each mutation was verified by sequencing. The other two vectors used in the system are pMDG encoding the vesicular stomatitis virus $G$ protein and retroviral expression vector pCSFLW which encodes for the luciferase reporter gene. Virus stocks were prepared by cotransfection of HEK293T cells as described previously [44-46], diluted 50- to 500-fold and used to infect HEK293T target cells. The virus and target cells were incubated with medium containing varying drug concentrations for $48 \mathrm{~h}$. Infectivity was determined by measuring luciferase activity in the target cells using Steady-Glo reporter assay system (Promega). Data were expressed relative to that of no drug controls and the drug concentrations required to inhibit virus replication by $50 \%\left(\mathrm{IC}_{50}\right)$ were determined by linear regression analysis. Results are expressed as fold changes in the $\mathrm{IC}_{50}$ compared to wild-type subtype $C$ virus.

\section{Antiretroviral drugs}

The NRTIs ABC, AZT, ddI, emtricitabine (FTC), 3TC and $\mathrm{d} 4 \mathrm{~T}$; and the NNRTIs efavirenz (EFV), etravirine (ETV), and NVP were obtained from the NIH AIDS Research and Reference Reagent Program. TDF was a generous gift from Gilead Sciences (Foster City, CA, USA).

\section{Replicative capacity Assay}

Recombinant viruses expressing wild-type and patientderived RT domains were normalized for p24 capsid (Genetic Systems HIV-1 Ag EIA; Bio-Rad) and used to infect target HEK293T cells in a single-cycle-replication assay. Replicative capacity was determined by measuring luciferase activity as described above.

\section{Statistical analyses}

Student's $t$ test was used to describe differences in $\mathrm{IC}_{50}$ values and replicative capacity and two proportions analysis was performed by using Fisher's Exact test with $P$ values $<0.05$ regarded as significant for both tests (StataSE software).

\section{Nucleotide sequence accession numbers}

The single-genome sequences generated and used in this study have been submitted to GenBank and assigned the accession numbers HQ111194-HQ111338.

\section{Acknowledgements}

We especially thank Sarah Palmer for technical advice in establishing the single-genome sequencing assay; Vinay Pathak, Stéphane Hué, and Andrew Buckton for helpful discussions; the patients, staff and project management of the CHAP2 cohort study in Lusaka, Zambia. We thank Nigel Temperton
University of Kent for pCSFLW; Didier Trono EPFL Switzerland for pCMV $\triangle 8.91$ and pMDG; and Thumbi Ndung'u, Boris Renjifo and Max Essex for p8MJ4. We also thank Soo-Yoon Rhee, Stanford University HIV database for help with database sequence analysis and Ross Harris, Health Protection Agency for help with statistical analysis.

This report is work financially supported by the National Institute for Health Research in Health Protection at the Health Protection Agency. The views expressed in this publication are those of the authors and not necessarily those of the NHS, the National Institute for Health Research or the Department of Health. DP is part funded by the NIHR UCLH/UCL Comprehensive Biomedical Research Centre and we acknowledge part funding from the UK Medical Research Council, the Wellcome Trust and the European Community's Seventh Framework Programme (FP7/2007-2013) under the project "Collaborative HIV and Anti-HIV Drug Resistance Network (CHAIN)" - grant agreement $n^{\circ} 223131$.

\section{Author details}

${ }^{1}$ Virus Reference Department, Microbiology Services, Colindale, Health Protection Agency, London, UK. ${ }^{2}$ UCL/MRC Centre for Medical Molecular Virology, Division of Infection and Immunity, UCL, Windeyer Institute, London, UK. ${ }^{3}$ University Teaching Hospital, UNZA School of Medicine, Lusaka, Zambia. ${ }^{4} \mathrm{MRC}$ Clinical Trials Unit, London, UK.

\section{Authors' contributions}

JLM carried out the bulk of the laboratory work, planning the study and writing the manuscript. RKG, CMP, DMG, ASW, PAC and DP were involved in planning the study, undertaking laboratory work and editing the manuscript. DK, VM, MK, CC, DMG were involved in undertaking clinical support work. All authors read and approved the final manuscript.

\section{Competing interests}

The authors declare that they have no competing interests.

Received: 3 January 2011 Accepted: 11 May 2011

Published: 11 May 2011

\section{References}

1. Panel on Antiretroviral Guidelines for Adults and Adolescents: Guidelines for the use of antiretroviral agents in HIV-1-infected adults and adolescents. Department of Health and Human Services 2009, 1-161, 20-72010.

2. Hammer SM, Eron JJ Jr, Reiss P, Schooley RT, Thompson MA, Walmsley S, et al: Antiretroviral treatment of adult HIV infection: 2008 recommendations of the International AIDS Society-USA panel. JAMA 2008, 300:555-570.

3. World Health Organization (WHO): Antiretroviral therapy for HIV infection in adults and adolescents: recommendations for a public health approach 2010, 20-7-2010.

4. Ren J, Stammers DK: HIV reverse transcriptase structures: designing new inhibitors and understanding mechanisms of drug resistance. Trends Pharmacol Sci 2005, 26:4-7.

5. Sarafianos SG, Das K, Ding J, Boyer PL, Hughes SH, Arnold E: Touching the heart of HIV-1 drug resistance: the fingers close down on the dNTP at the polymerase active site. Chem Biol 1999, 6:R137-R146.

6. Kohlstaedt LA, Wang J, Friedman JM, Rice PA, Steitz TA: Crystal structure at 3.5 A resolution of HIV-1 reverse transcriptase complexed with an inhibitor. Science 1992, 256:1783-1790.

7. Huang H, Chopra R, Verdine GL, Harrison SC: Structure of a covalently trapped catalytic complex of HIV-1 reverse transcriptase: implications for drug resistance. Science 1998, 282:1669-1675.

8. Menendez-Arias L, Martinez MA, Quinones-Mateu ME, Martinez-Picado J: Fitness variations and their impact on the evolution of antiretroviral drug resistance. Curr Drug Targets Infect Disord 2003, 3:355-371.

9. Harada S, Hazra R, Tamiya S, Zeichner SL, Mitsuya H: Emergence of human immunodeficiency virus type 1 variants containing the Q151M complex in children receiving long-term antiretroviral chemotherapy. Antiviral Res 2007, 75:159-166.

10. Shafer RW, Schapiro JM: HIV-1 drug resistance mutations: an updated framework for the second decade of HAART. AIDS Rev 2008, 10:67-84.

11. Shirasaka T, Kavlick MF, Ueno T, Gao WY, Kojima E, Alcaide ML, et al: Emergence of human immunodeficiency virus type 1 variants with 
resistance to multiple dideoxynucleosides in patients receiving therapy with dideoxynucleosides. Proc Natl Acad Sci USA 1995, 92:2398-2402.

12. Iversen AK, Shafer RW, Wehrly K, Winters MA, Mullins JI, Chesebro B, et al: Multidrug-resistant human immunodeficiency virus type 1 strains resulting from combination antiretroviral therapy. J Virol 1996, 70:1086-1090.

13. Garcia-Lerma JG, Gerrish PJ, Wright AC, Qari SH, Heneine W: Evidence of a role for the Q151L mutation and the viral background in development of multiple dideoxynucleoside-resistant human immunodeficiency virus type 1. J Virol 2000, 74:9339-9346

14. Kosalaraksa P, Kavlick MF, Maroun V, Le R, Mitsuya H: Comparative fitness of multi-dideoxynucleoside-resistant human immunodeficiency virus type 1 (HIV-1) in an In vitro competitive HIV-1 replication assay. J Virol 1999, 73:5356-5363.

15. Matsumi S, Kosalaraksa P, Tsang H, Kavlick MF, Harada S, Mitsuya H: Pathways for the emergence of multi-dideoxynucleoside-resistant HIV-1 variants. AIDS 2003, 17:1127-1137.

16. Hosseinipour MC, van Oosterhout JJ, Weigel R, Phiri S, Kamwendo D, Parkin N, et al: The public health approach to identify antiretroviral therapy failure: high-level nucleoside reverse transcriptase inhibitor resistance among Malawians failing first-line antiretroviral therapy. AIDS 2009, 23:1127-1134.

17. Sirivichayakul S, Ruxrungtham K, Ungsedhapand C, Techasathit W, Ubolyam S, Chuenyam T, et al: Nucleoside analogue mutations and Q151M in HIV-1 subtype A/E infection treated with nucleoside reverse transcriptase inhibitors. AIDS 2003, 17:1889-1896.

18. Sungkanuparph S, Manosuthi W, Kiertiburanakul S, Piyavong B, Chumpathat N, Chantratita W: Options for a second-line antiretroviral regimen for HIV type 1-infected patients whose initial regimen of a fixed-dose combination of stavudine, lamivudine, and nevirapine fails. Clin Infect Dis 2007, 44:447-452.

19. Gupta RK, Ford D, Mulenga V, Walker AS, Kabamba D, Kalumbi M, et al: Drug Resistance in Human Immunodeficiency Virus Type-1 Infected Zambian Children Using Adult Fixed Dose Combination Stavudine, Lamivudine, and Nevirapine. Pediatr Infect Dis J 2010.

20. Delviks-Frankenberry KA, Nikolenko GN, Pathak VK: The "Connection" Between HIV Drug Resistance and RNase H. Viruses 2010, 2:1476-1503.

21. Ehteshami M, Gotte M: Effects of mutations in the connection and RNase $\mathrm{H}$ domains of HIV-1 reverse transcriptase on drug susceptibility. AIDS ReV 2008, 10:224-235.

22. Yap SH, Sheen CW, Fahey J, Zanin M, Tyssen D, Lima VD, et al: N348I in the connection domain of HIV-1 reverse transcriptase confers zidovudine and nevirapine resistance. PLoS Med 2007, 4:e335.

23. Delviks-Frankenberry KA, Nikolenko GN, Boyer PL, Hughes SH, Coffin JM, Jere $A$, et al: HIV-1 reverse transcriptase connection subdomain mutations reduce template RNA degradation and enhance AZT excision. Proc Natl Acad Sci USA 2008, 105:10943-10948.

24. Delviks-Frankenberry KA, Nikolenko GN, Barr R, Pathak VK: Mutations in human immunodeficiency virus type 1 RNase $\mathrm{H}$ primer grip enhance $3^{\prime}$ azido-3'-deoxythymidine resistance. J Virol 2007, 81:6837-6845.

25. Nikolenko GN, Palmer S, Maldarelli F, Mellors JW, Coffin JM, Pathak VK: Mechanism for nucleoside analog-mediated abrogation of HIV-1 replication: balance between RNase $\mathrm{H}$ activity and nucleotide excision. Proc Natl Acad Sci USA 2005, 102:2093-2098.

26. Ehteshami M, Beilhartz GL, Scarth BJ, Tchesnokov EP, McCormick S, Wynhoven B, et al: Connection domain mutations N348I and A360V in HIV-1 reverse transcriptase enhance resistance to 3 '-azido-3'deoxythymidine through both RNase $\mathrm{H}$-dependent and -independent mechanisms. J Biol Chem 2008, 283:22222-22232.

27. Gotte M: Should we include connection domain mutations of HIV-1 reverse transcriptase in HIV resistance testing. PLoS Med 2007, 4:e346.

28. Gallego O, Mendoza C, Labarga P, Altisent C, Gonzalez J, Garcia-Alcalde I, et al: Long-term outcome of HIV-infected patients with multinucleosideresistant genotypes. HIV Clin Trials 2003, 4:372-381.

29. Johnson VA, Brun-Vezinet F, Clotet B, Gunthard HF, Kuritzkes DR, Pillay D, et al: Update of the drug resistance mutations in HIV-1: December 2010. Top HIV Med 2010, 18:156-163.

30. Petropoulos CJ, Parkin NT, Limoli KL, Lie YS, Wrin T, Huang W, et al: A novel phenotypic drug susceptibility assay for human immunodeficiency virus type 1. Antimicrob Agents Chemother 2000, 44:920-928.
31. Barnas D, Koontz D, Bazmi H, Bixby C, Jemsek J, Mellors JW: Clonal resistance analyses of HIV type-1 after failure of therapy with didanosine, lamivudine and tenofovir. Antivir Ther 2010, 15:437-441.

32. Delviks-Frankenberry KA, Nikolenko GN, Maldarelli F, Hase S, Takebe $Y$, Pathak VK: Subtype-specific differences in the human immunodeficiency virus type 1 reverse transcriptase connection subdomain of CRF01_AE are associated with higher levels of resistance to 3'-azido-3'deoxythymidine. J Virol 2009, 83:8502-8513.

33. Wolf $\mathrm{K}$, Walter $\mathrm{H}$, Beerenwinkel $\mathrm{N}$, Keulen $\mathrm{W}$, Kaiser $\mathrm{R}$, Hoffmann $\mathrm{D}$, et al: Tenofovir resistance and resensitization. Antimicrob Agents Chemother 2003, 47:3478-3484.

34. Miller MD, Margot NA, Hertogs K, Larder B, Miller V: Antiviral activity of tenofovir (PMPA) against nucleoside-resistant clinical HIV samples. Nucleosides Nucleotides Nucleic Acids 2001, 20:1025-1028.

35. Back NK, Nijhuis M, Keulen W, Boucher CA, Oude Essink BO, van Kuilenburg AB, et al: Reduced replication of 3TC-resistant HIV-1 variants in primary cells due to a processivity defect of the reverse transcriptase enzyme. EMBO J 1996, 15:4040-4049.

36. Wei X, Liang C, Gotte M, Wainberg MA: Negative effect of the M184V mutation in HIV-1 reverse transcriptase on initiation of viral DNA synthesis. Virology 2003, 311:202-212.

37. Geretti AM: HIV-1 subtypes: epidemiology and significance for HIV management. Curr Opin Infect Dis 2006, 19:1-7.

38. Shafer RW, Winters MA, Iversen AK, Merigan TC: Genotypic and phenotypic changes during culture of a multinucleoside-resistant human immunodeficiency virus type 1 strain in the presence and absence of additional reverse transcriptase inhibitors. Antimicrob Agents Chemother 1996, 40:2887-2890.

39. Maeda Y, Venzon DJ, Mitsuya H: Altered drug sensitivity, fitness, and evolution of human immunodeficiency virus type 1 with pol gene mutations conferring multi-dideoxynucleoside resistance. J Infect Dis 1998, 177:1207-1213

40. Palmer S, Kearney M, Maldarelli F, Halvas EK, Bixby CJ, Bazmi H, et al: Multiple, linked human immunodeficiency virus type 1 drug resistance mutations in treatment-experienced patients are missed by standard genotype analysis. J Clin Microbiol 2005, 43:406-413.

41. Tamura K, Dudley J, Nei M, Kumar S: MEGA4: Molecular Evolutionary Genetics Analysis (MEGA) software version 4.0. Mol Biol Evol 2007, 24:1596-1599.

42. Parry CM, Kohli A, Boinett CJ, Towers GJ, McCormick AL, Pillay D: Gag determinants of fitness and drug susceptibility in protease inhibitorresistant human immunodeficiency virus type 1. J Virol 2009, 83:9094-9101.

43. Gupta RK, Kohli A, McCormick AL, Towers GJ, Pillay D, Parry CM: Full-length HIV-1 Gag determines protease inhibitor. AIDS 2010, 24:1651-1655.

44. Bainbridge JW, Stephens C, Parsley K, Demaison C, Halfyard A, Thrasher AJ, et al: In vivo gene transfer to the mouse eye using an HIV-based lentiviral vector; efficient long-term transduction of corneal endothelium and retinal pigment epithelium. Gene Ther 2001, 8:1665-1668.

45. Besnier C, Takeuchi Y, Towers G: Restriction of lentivirus in monkeys. Proc Natl Acad Sci USA 2002, 99:11920-11925.

46. Wright E, Temperton NJ, Marston DA, McElhinney LM, Fooks AR, Weiss RA: Investigating antibody neutralization of lyssaviruses using lentiviral pseudotypes: a cross-species comparison. J Gen Virol 2008, 89:2204-2213.

doi:10.1186/1742-4690-8-31

Cite this article as: Mbisa et al:: The evolution of HIV-1 reverse transcriptase in route to acquisition of Q151M multi-drug resistance is complex and involves mutations in multiple domains. Retrovirology 2011 8:31. 